\title{
Study of Confinement/Deconfinement Transition in AdS/QCD with Generalized Warp Factors
}

\author{
Shobhit Sachan \\ Department of Physics, Banaras Hindu University, Varanasi 221005, India \\ Correspondence should be addressed to Shobhit Sachan; shobhitsachan@gmail.com
}

Received 27 June 2014; Accepted 13 August 2014; Published 21 August 2014

Academic Editor: George Siopsis

Copyright (C) 2014 Shobhit Sachan. This is an open access article distributed under the Creative Commons Attribution License, which permits unrestricted use, distribution, and reproduction in any medium, provided the original work is properly cited. The publication of this article was funded by SCOAP S $^{3}$

\begin{abstract}
We study analytical solutions of charged black holes and thermally charged AdS with generalized warped factors in EinsteinMaxwell-Dilaton system. We calculate Euclidean action for charged AdS and thermally charged AdS. The actions in both backgrounds are regularized by the method of background subtraction. The study of phase transition between charged black hole and thermally charged AdS gives an insight into the confinement/deconfinement transition. The plots of grand potential versus temperature and chemical potential versus transition temperature are obtained.
\end{abstract}

\section{Introduction}

Strongly interacting systems are always a challenge to our analytical knowledge. Quantum chromodynamics is such a theory, which cannot be solved analytically in low energy regime. There are two methods to solve QCD; one is "lattice QCD" [1] and the other is "AdS/QCD." The formulation of lattice QCD is based on discretization of spacetime and it requires high performance computing. On the other hand, AdS/QCD is analytic approach and is motivated by the gauge/gravity duality [2-5]. Some properties of QCD like theories motivated by gauge/gravity duality such as confinement and chiral symmetry breaking have been studied extensively in [6-21] and spectrum of mesons and baryons is studied in $[22,23]$.

There are two approaches from where one can construct QCD like theories. These approaches are known as top-down and bottom-up approaches. In top-down approach, one starts from stringy $\mathrm{D}$ brane configurations and constructs models for QCD [24, 25] while in bottom-up approach, one starts from QCD and attempts to construct its five-dimensional gravity dual. These five-dimensional dual models can be generalized to study various properties of QCD. The bottom-up approach is divided into two categories, hard wall [7] and soft wall models [8]. In hard wall model, one imposes a cutoff at
IR boundary. The IR cutoff in hard wall model is inverse of the QCD scale. The hard wall model describes many properties of QCD such as form factors, effective coupling constants, chiral symmetry breaking, and correlation functions but fails to accommodate Regge trajectory of meson masses. The problem of mass spectra can be removed by introduction of a dilaton field. This model is known as soft wall model of AdS/QCD and the IR boundary in this model is shifted to infinity.

The transition between confining and deconfining phase is studied by Hawking-Page transition in bulk spacetime [26]. The high temperature phase is charged AdS black hole while low temperature phase is thermally charged AdS geometry. The confinement/deconfinement is studied in hard wall and soft wall models in [14] and models with chemical potential are studied in [19-21,27].

In the charged black hole solutions, charge of black hole is related to chemical potential of the quarks. The dual gauge theory defining the deconfining phase is AdS black hole while the confining phase is defined by thermally charged AdS solutions [21]. The UV divergences in these actions are removed by subtraction of action of thermal AdS $[19,21,28]$.

The study of gauge/gravity duality provides a relation between the gravity theories in the AdS spacetime and conformal field theories on the boundary of the AdS spacetime. 
In recent years, a large number of generalized geometries are studied, which gives a dual scale invariant gauge theory. One of the metrics representing such a geometry is given by

$$
\begin{array}{r}
d s^{2}=r^{2 \alpha}\left(r^{2 z} f(r) d t^{2}+\frac{1}{r^{2} f(r)} d r^{2}+r^{2} d \vec{x}^{2}\right), \\
\text { where } \alpha=-\frac{\theta}{d} .
\end{array}
$$

In this metric, the constants $z$ and $\theta$ are dynamical and hyperscaling violation exponents, respectively. This metric gives AdS solutions for $\theta=0$ and $z=1$. The scale transformations $t \rightarrow \lambda^{z}, r \rightarrow \lambda^{-1} r, x_{i} \rightarrow \lambda x_{i}$ lead to $d s_{d+2}^{2} \rightarrow \lambda^{\theta / d} d s_{d+2}^{2}$. Thus, the transformations retain the spatially homogeneous and covariant nature, but the distance scales as powers of $\lambda$ for nonzero values of $\theta$. The noninvariance of distance in reference of AdS/CFT correspondence leads to violation of hyperscaling in dual field theory. In other words, it is shown in [29] that, if the hyperscaling violation exponent is included in the metric, the entropy scales as $T^{(d-\theta) / z}$. If hyperscaling is not taken into account, the entropy scales as $T^{d / z}$ [29-31].

In this paper, we study the effect of warping on confinement/deconfinement transition in the simplest case by taking the value of $z=1$. In this context, we have only single charge in AdS black hole [32]. We first study the holographic model of QCD with a dilaton potential in Einstein-Maxwell-Dilaton system. The form of potential is taken to be exponential with some free parameters. These parameters are fixed by various boundary conditions. We calculate Euclidean actions of charged AdS black hole and thermally charged AdS. These actions are regularized by subtraction of thermal AdS action. The Hawking-Page transition is studied and plots between grand potential versus temperature and transition temperature versus chemical potential are given. The plots of transition temperature $\left(T_{c}\right)$ versus chemical potential $\mu$ in Figure 2 are plotted for different values of $\alpha$. The warp factor $\alpha$ depends on hyperscaling violation exponent; therefore, the transition temperature varies with hyperscaling violation exponent. The transition temperature becomes independent of warp factor (or hyperscaling violation exponent) for certain value of $\mu$. This point, where all curves meet, may be a signature of second-order transition.

This paper is organized in six sections. In Section 2, we briefly summarized the calculations for $z=1$ and single gauge field. In Sections 3 and 4, grand potentials are calculated for charged AdS black hole and thermally charged AdS. Section 5 is devoted to the study of confinement/deconfinement transition and in Section 6 we conclude our analysis.

\section{Gravitational Solution of EMD Theory}

In this section the solution Einstein-Maxwell-Dilaton system with hyperscaling violation [32] is given. We take the solution obtained in [32] and take dynamical exponent $z=1$. Taking $z=1$ limits the number of gauge fields in solution to one.
We begin with well-known Einstein-Maxwell-Dilaton action with exponential potential $\left(V=V_{0} e^{\gamma \phi}\right)$ given by

$$
\begin{aligned}
S=-\int & d^{d+2} x \sqrt{g} \\
& \times\left[\frac{1}{2 \kappa^{2}}\left(R-\frac{1}{2}(\partial \phi)^{2}+V_{0} e^{\gamma \phi}\right)-\frac{1}{4 g^{2}} F^{2} e^{\lambda \phi}\right],
\end{aligned}
$$

where $\kappa^{2}=8 \pi G, g$ is coupling constant of dimension $d+2$, and $\lambda, \gamma$, and $V_{0}$ are parameters of the model, which will be fixed later.

The equations of motion for gravitational part of action (2) can be written as

$$
\frac{1}{2 \kappa^{2}}\left\{G_{\mu \nu}-\frac{1}{2}\left(-\frac{g_{\mu \nu}}{2}(\partial \phi)^{2}+\partial_{\mu} \phi \partial_{\nu} \phi\right)-\frac{1}{2} g_{\mu \nu} V_{0} e^{\gamma \phi}\right\}=T_{\mu \nu}
$$

where

$$
G_{\mu \nu}=R_{\mu \nu}-\frac{1}{2} g_{\mu \nu} R, \quad T_{\mu \nu}=\frac{1}{2 g^{2}} e^{\lambda \phi}\left\{F_{\mu}^{\rho} F_{\rho \nu}-\frac{1}{4} F^{2} g_{\mu \nu}\right\} .
$$

The equations of motion (3) can be written in modified form as

$$
R_{\mu \nu}+\frac{V_{0} e^{\gamma \phi}}{d} g_{\mu \nu}=\frac{1}{2} \partial_{\mu} \phi \partial_{\nu} \phi+\frac{\kappa^{2}}{g^{2}} e^{\lambda \phi}\left\{F_{\mu}^{\rho} F_{\rho \nu}-\frac{1}{2 d} F^{2} g_{\mu \nu}\right\} .
$$

The equation of motion for scalar field is

$$
\frac{1}{\sqrt{g}} \partial_{\mu}\left(\sqrt{g} g^{\mu \nu} \partial_{\nu} \phi\right)=-\frac{\partial\left(V_{0} e^{\gamma \phi}\right)}{\partial \phi}+\frac{1}{2} \frac{\kappa^{2}}{g^{2}} \lambda e^{\lambda \phi} F^{2}
$$

and for gauge field is

$$
\frac{1}{\sqrt{g}} \partial_{\mu}\left(\sqrt{g} e^{\lambda \phi} F^{\mu \nu}\right)=0 .
$$

Let us consider the ansatz for our metric (with $z=1$ ), scalar field, and gauge field, which are given as

$$
\begin{gathered}
d s^{2}=r^{2 \alpha}\left(r^{2} f(r) d t^{2}+\frac{1}{r^{2} f(r)} d r^{2}+r^{2} d \vec{x}^{2}\right), \\
\phi=\phi(r), F_{r, t} \neq 0 .
\end{gathered}
$$

We consider $F_{\mu \nu}$ as the only function of $r$ and the rest of the components are equal to zero.

Using our ansatz, the solution for Maxwell's equations can be written as

$$
F_{r t}=e^{-\lambda \phi} r^{\alpha(2-d)-d} \rho,
$$

where $\rho$ is integration constant and is to be related to charge of the black hole later.

On solving $t t$ and $r r$ components of Einstein's equations, we determine the scalar field, which is given as

$$
e^{\phi}=e^{\phi_{0}} r^{\sqrt{2 d \alpha(\alpha+1)}}=e^{\phi_{0}} r^{\zeta} \text {. }
$$


The exponent on $r$ shows that, to get well-defined solutions, we must have $\alpha(\alpha+1) \geq 0$. by

Using equations of motion, the metric function is given

$$
f(r)=1-\frac{m}{r^{d(1+\alpha)+1}}+\frac{Q^{2}}{r^{2 d(1+\alpha)}},
$$

where $m$ is related to the mass of the black hole and $Q$ is related to $\rho$ by the following relation:

$$
Q^{2}=-\frac{\kappa^{2}}{g^{2}} \frac{e^{-\lambda \phi_{0}}}{d(1+\alpha)(-1+d-2 \alpha+d \alpha+\zeta \lambda)} \rho^{2} .
$$

The parameter $\gamma$ appearing in exponential of potential is fixed by using the fact that the a constant term (independent of $r$ ) appears in metric. Equating the powers of $r$ to zero, the parameter $\gamma$ can be fixed as $-2 \alpha / \zeta$. The constant term is equated to unity to get the value $V_{0}$, which is given by relation

$$
V_{0}=d(1+\alpha)(1+d+d \alpha) e^{-\gamma \phi_{0}},
$$

and using equation of motion for scalar field, the value of parameter $\lambda$ is fixed as $-\gamma$. The solution for field strength (9) becomes

$$
F_{r t}=i \bar{Q} r^{-d(\alpha+1)}
$$

where we have defined $\bar{Q}=(g / \kappa) Q \sqrt{d(1+\alpha)(d \alpha+d-1)}$ $e^{-\lambda \phi_{0} / 2}$. The solution of gauge field $A_{t}$ is given by

$$
A_{t}(r)=\frac{i \bar{Q}}{1-d(\alpha+1)} r^{-d(\alpha+1)+1}+C,
$$

where $C$ is a constant and related to boundary value of $A_{t}$, which is chemical potential of the system.

\section{AdS Black Hole}

In this section, we consider the black hole solution for the warped geometry. The solution of $A_{t}$ with appropriate boundary conditions leads us to the solution of charged AdS black hole. Using the solution of $A_{t}$ in (15), we apply the condition that, at the boundary $(r \rightarrow \infty)$, the value of $A_{t}$ is $i \mu$, where $\mu$ is chemical potential of the black hole and $i$ is due to the consideration of Euclidean signature. The boundary value gives us the constant $C=i \mu$ and the solution of $A_{t}$ has the form

$$
A_{t}(r)=i\left(\mu-\frac{\bar{Q}}{d(\alpha+1)-1} r^{-d(\alpha+1)+1}\right) .
$$

At horizon $\left(r_{H}\right), A_{t}=0$ leads us to the relation between $\mu$ and $\bar{Q}$, which is given by

$$
\begin{aligned}
\bar{Q} & =\frac{d(\alpha+1)-1}{r_{H}^{-d(\alpha+1)+1}} \mu, \\
\Longrightarrow Q & =\frac{\kappa}{g} \mu \sqrt{\frac{d(1+\alpha)-1}{d(1+\alpha)}} e^{\lambda \phi_{0} / 2} r_{H}^{d(\alpha+1)-1} .
\end{aligned}
$$

The radius of horizon for charged black hole solution is obtained by equating the metric function $f\left(r_{H}\right)=0$. This leads to the equation for $r_{H}$, which is given as

$$
r_{H}^{2 d(1+\alpha)}-m r_{H}^{d(1+\alpha)-1}+Q^{2}=0,
$$

and the Hawking temperature of the black hole is given by

$$
\begin{aligned}
T= & \frac{1}{4 \pi}(d \alpha+d+1) r_{H}\left(1-Q^{2} \frac{d \alpha+d-1}{d \alpha+d+1} r_{H}^{-2 d(1+\alpha)}\right) \\
= & \frac{1}{4 \pi}(d \alpha+d+1) r_{H} \\
& \times\left(1-\mu^{2} \frac{\kappa^{2}}{g^{2}} \frac{(d \alpha+d-1)^{2}}{d(1+\alpha)(d \alpha+d+1)} e^{\lambda \phi_{0}} \frac{1}{r_{H}^{2}}\right) .
\end{aligned}
$$

Now redefining some variables for simplicity,

$$
D_{1}=\frac{d \alpha+d+1}{4 \pi}, \quad D_{2}=\frac{\kappa^{2}}{g^{2}} \frac{(d \alpha+d-1)^{2}}{d(1+\alpha)(d \alpha+d+1)} e^{\lambda \phi_{0}} .
$$

Using these redefinitions in (19) and solving the quadratic equation, we get positive value of horizon radius as

$$
r_{H}=\frac{T+\sqrt{T^{2}+4 \mu^{2} D_{1}^{2} D_{2}}}{2 D_{1}} .
$$

Using equation of motion, action (2) can be written as

$$
\begin{aligned}
S^{\mathrm{AdSBH}} & =\frac{1}{d} \int d^{d+2} x \sqrt{g}\left[\frac{V}{\kappa^{2}}+\frac{1}{2 g^{2}} e^{\lambda \phi} F^{2}\right] \\
& =\frac{1}{d} V_{d} \beta \int d r \sqrt{g}\left[\frac{V}{\kappa^{2}}+\frac{1}{2 g^{2}} e^{\lambda \phi} F^{2}\right],
\end{aligned}
$$

where $V_{d}$ is $d$-dimensional volume and $\beta$ is inverse of black hole temperature. On substituting various values in the above action, it simplifies to

$$
\begin{gathered}
S^{\mathrm{AdSBH}}=\frac{1}{d} V_{d} \beta \frac{d(1+\alpha)}{\kappa^{2}} \int d r r^{d(\alpha+1)+2 \alpha} \\
\times\left[(d \alpha+d+1) r^{-2 \alpha}\right. \\
\left.-Q^{2}(d \alpha+d-1) r^{-2 d(\alpha+1)-2 \alpha}\right] \\
=\frac{1}{d} V_{d} \beta \frac{d(1+\alpha)}{\kappa^{2}}\left[r^{d(\alpha+1)+1}+Q^{2} r^{-d(\alpha+1)-1}\right]_{r_{H}}^{r_{\max }},
\end{gathered}
$$

where we take $r_{\max } \rightarrow \infty$ at the end of the calculations.

The above action is singular at $r_{\max } \rightarrow \infty$. Therefore, to regularize this action we subtract thermal AdS from this action. The metric for thermal AdS is given by

$$
d s^{2}=r^{2 \alpha}\left(r^{2} d t^{2}+\frac{1}{r^{2}} d r^{2}+r^{2} d \vec{x}^{2}\right),
$$


and action for thermal AdS with time periodicity $\beta_{1}$ is given by equation

$$
S^{\mathrm{tAdS}}=-V_{d} \beta_{1} \frac{(1+\alpha)}{\kappa^{2}}\left(r_{\max }\right)^{d(\alpha+1)+1} .
$$

Thus the regularized action for AdS black hole is given by

$$
\begin{aligned}
\bar{S}^{\mathrm{AdSBH}}= & \lim _{r_{\max } \rightarrow \infty} V_{d} \beta \frac{(1+\alpha)}{\kappa^{2}} \\
& \times\left\{\left[r^{d(\alpha+1)+1}+Q^{2} r^{-d(\alpha+1)-1}\right]_{r_{H}}^{r_{\max }}\right. \\
& \left.\quad-\left.\left(\frac{f\left(r_{\max }\right)}{f\left(r_{\max }, m=Q=0\right)}\right)^{1 / 2} r^{d(\alpha+1)+1}\right|_{0} ^{r_{\max }}\right\} \\
= & -V_{d} \beta \frac{(1+\alpha)}{\kappa^{2}}\left(\left(r_{H}\right)^{d(\alpha+1)+1}+Q^{2}\left(r_{H}\right)^{-d(\alpha+1)+1}\right) .
\end{aligned}
$$

The factor $\left(f\left(r_{\max }\right) / f\left(r_{\max }, m=Q=0\right)\right)^{1 / 2}$ in front of the last term in the first expression is inserted to match the Euclidean time periodicity at $r=r_{\max }$, where both the solutions coincide with each other. The singular term (powers of $r$ with positive values) of AdS black hole solution is cancelled with the term in thermal AdS solution and we get the regularized action. By using thermodynamical relation, $\Omega(\mu, T)=T S_{\text {on-shell }}$, we write the regularized grand potential for AdS black hole as

$$
\Omega^{\mathrm{AdSBH}}=-V_{d} \frac{1+\alpha}{\kappa^{2}}\left(\left(r_{H}\right)^{d(\alpha+1)+1}+\left(r_{H}\right)^{-d(\alpha+1)+1} Q^{2}\right) .
$$

\section{Thermally Charged AdS}

This section is devoted to the study of thermally charged AdS solution [21]. The thermally charged AdS is also asymptotically AdS but does not have a horizon. Due to absence of horizon, we choose a lower cutoff for thermally charged AdS as $r_{\mathrm{IR}}$ and integrate from $r_{\mathrm{IR}}$ to $\infty$. The metric function for thermally charged AdS is given by

$$
f_{1}(r)=1+\frac{Q_{1}^{2}}{r^{2 d(\alpha+1)}},
$$

where $Q_{1}$ is charge associated with thermally charged AdS. This metric function also satisfies Einstein-Maxwell equations. This geometry is simply obtained by putting $m=0$ in solution of AdS black hole. The charge $Q_{1}$ in this case is different from that of AdS black hole due to different boundary conditions.

The field strength tensor for thermally charged AdS is given by the same equation as that for AdS black hole case, but now $Q$ is replaced by $Q_{1}$. Th expression is written as

$$
F_{1 r t}=i \bar{Q}_{1} r^{-d(\alpha+1)},
$$

$$
\text { where } \bar{Q}_{1}=\frac{g}{\kappa} Q_{1} \sqrt{d(\alpha+1)(d \alpha+d-1)} e^{-\lambda \phi_{0} / 2} \text {. }
$$

From this field strength, the gauge field can be calculated as

$$
A_{1 t}(r)=\frac{i \bar{Q}_{1}}{1-d(\alpha+1)} r^{-d(\alpha+1)+1}+C_{2} .
$$

Again at $r \rightarrow \infty$, we have $A_{1 t}(\infty)=C_{2}=i \mu$, but at $r=r_{\mathrm{IR}}$, we apply Dirichlet boundary condition $A_{1 t}\left(r_{\mathrm{IR}}\right)=i \xi \mu$, where $\xi$ is a constant to determined. Thus, at $r_{\mathrm{IR}}$,

$$
\begin{aligned}
& A_{1 t}\left(r_{\mathrm{IR}}\right)=i \xi \mu=i \mu-\frac{i \overline{\mathrm{Q}}_{1}}{d(\alpha+1)-1} r^{-d(\alpha+1)+1} \\
& \quad \Longrightarrow Q_{1}=\frac{\kappa}{g} \mu(1-\xi) \sqrt{\frac{d(\alpha+1)-1}{d(\alpha+1)}} e^{\lambda \phi_{0} / 2} r_{\mathrm{IR}}^{d(\alpha+1)-1} .
\end{aligned}
$$

Using the same procedure as done for AdS black hole, we compute regularized action for thermally charged AdS, which is written as

$$
\begin{aligned}
\bar{S}^{\text {tcAdS }}= & \lim _{r_{\max } \rightarrow \infty} V_{d} \beta_{1} \frac{(1+\alpha)}{\kappa^{2}} \\
& \times\left\{\left[r^{d(\alpha+1)+1}+Q_{1}^{2} r^{-d(\alpha+1)-1}\right]_{r_{\mathrm{IR}}}^{r_{\max }}\right. \\
& \left.-\left.\left(\frac{f_{1}\left(r_{\max }\right)}{f_{1}\left(r_{\max }, Q_{1}=0\right)}\right)^{1 / 2} r^{d(\alpha+1)+1}\right|_{0} ^{r_{\max }}\right\} \\
= & -V_{d} \beta_{1} \frac{(1+\alpha)}{\kappa^{2}}\left(\left(r_{\mathrm{IR}}\right)^{d(\alpha+1)+1}+Q_{1}^{2}\left(r_{\mathrm{IR}}\right)^{-d(\alpha+1)+1}\right),
\end{aligned}
$$

and grand potential for thermally charged AdS is given by equation

$$
\Omega^{\mathrm{tcAdS}}=-V_{d} \frac{1+\alpha}{\kappa^{2}}\left(\left(r_{\mathrm{IR}}\right)^{d(\alpha+1)+1}+Q_{1}^{2}\left(r_{\mathrm{IR}}\right)^{-d(\alpha+1)+1}\right),
$$

where $Q_{1}$ is function of chemical potential $\mu$. Using thermodynamical relation $N=-\partial \Omega / \partial \mu$, we calculate the quark number for thermally charged AdS, which is given by relation

$$
N=2 \frac{(\alpha+1)}{d g^{2}} \mu(1-\xi)^{2}(d(\alpha+1)-1) e^{\lambda \phi_{0}} r_{\mathrm{IR}}^{d(\alpha+1)-1} .
$$

As shown in [21], one has to use Dirichlet boundary condition instead of Neumann, we get free energy from Legendre transformation of grand potential, and $\mu N \beta_{1}$ is equal to boundary action $S^{\text {tcAdS }}$. Calculating the boundary action, we can determine unknown parameter $\xi$. The boundary action of thermally charged AdS is given by

$$
\begin{aligned}
S_{b}^{\mathrm{tcAdS}} & =\frac{1}{d} \int_{\partial M} d^{d+1} x \sqrt{g^{(d+1)}} \eta^{\sigma} A_{\rho} F_{\mu \sigma} g^{\rho \mu} e^{\lambda \phi} \\
& =\frac{1}{d g^{2}} V_{d} \beta_{1} \mu \bar{Q}_{1} e^{\lambda \phi},
\end{aligned}
$$

where unit vector $\eta^{r}=\left(0,-\sqrt{f_{1}(r)} / r^{\alpha-1}, 0,0 \ldots\right)$ and $g^{d+1}=$ $r^{(\alpha+1)(d+1)} \sqrt{f_{1}(r)}$. Comparing (34) and (35), we evaluated 


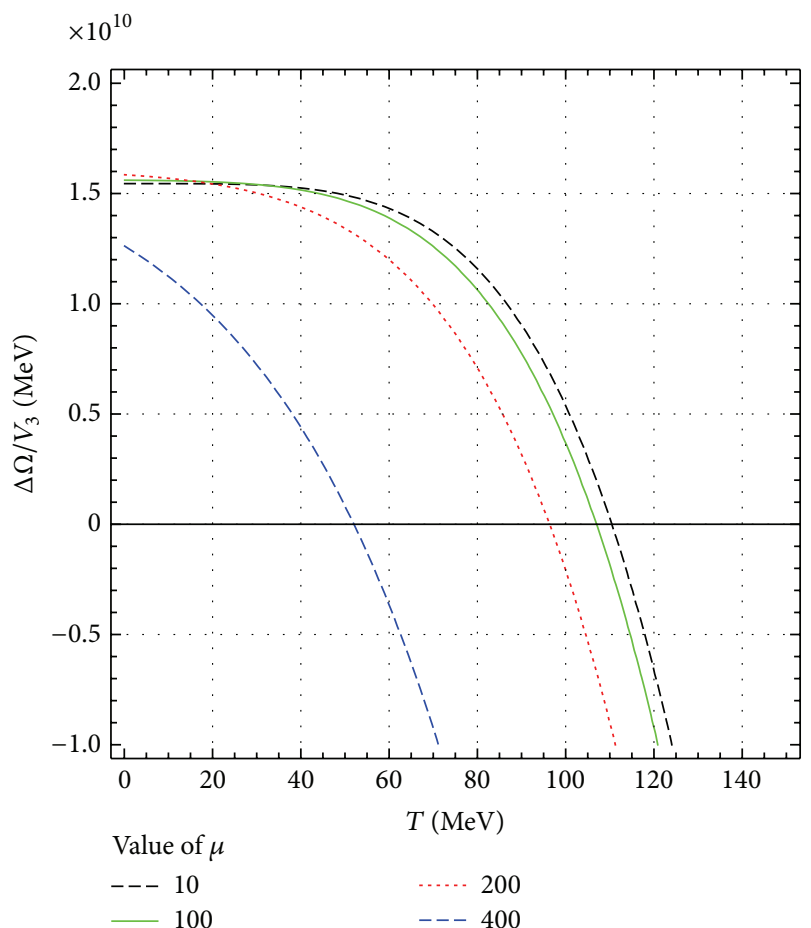

(a) For $\alpha=0.1$

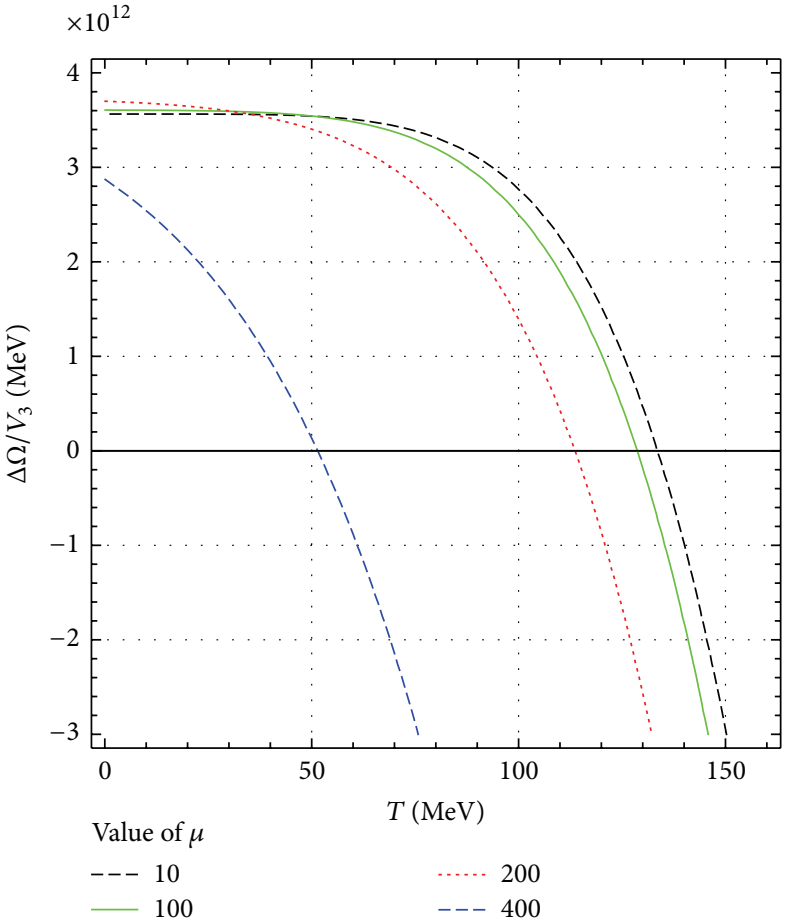

(b) For $\alpha=0.3$

FIgURE 1: Grand potential versus temperature at various values of $\mu$ for constant $\alpha$.

constant $\xi=1 / 2$. Thus charge $Q_{1}$ of thermally charged AdS is given by

$$
Q_{1}=\frac{\kappa}{2 g} \mu \sqrt{\frac{d(\alpha+1)-1}{d(\alpha+1)}} e^{\lambda \phi_{0} / 2} r_{\mathrm{IR}}^{d(\alpha+1)-1} .
$$

\section{Confinement/Deconfinement Transition}

Now we study the transition from AdS black hole phase to thermally charged AdS. To study this, we take the difference between the actions of AdS black hole and thermally charged AdS geometries with appropriate periodicity matching. The difference in grand potentials is proportional to difference in actions. The difference in actions is given by

$$
\begin{aligned}
& \Delta S=\lim _{r_{\max } \rightarrow \infty} \frac{1}{d} V_{d} \beta \frac{d(1+\alpha)}{\kappa^{2}} \\
& \times\left\{\left[r^{d(\alpha+1)+1}+Q^{2} r^{-d(\alpha+1)-1}\right]_{r_{H}}^{r_{\max }}\right. \\
&\left.\quad-\left(\frac{f\left(r_{\max }\right)}{f_{1}\left(r_{\max }\right)}\right)^{1 / 2}\left[r^{d(\alpha+1)+1}+Q_{1}^{2} r^{-d(\alpha+1)-1}\right]_{r_{\mathrm{IR}}}^{r_{\max }}\right\} \\
&=V_{d} \beta \frac{1}{\kappa^{2}}\left\{\left(\left(r_{\mathrm{IR}}\right)^{d(\alpha+1)+1}-\left(r_{H}\right)^{d(\alpha+1)+1}\right)\right. \\
& \quad+\mu^{2}\{d(1+\alpha)-1\} e^{\lambda \phi_{0}} \\
&\left.\times\left(\frac{1}{4} r_{\mathrm{IR}}^{d(\alpha+1)-1}-r_{H}^{d(\alpha+1)-1}\right)\right\} .
\end{aligned}
$$

The factor $\left(f\left(r_{\max }\right) / f_{1}\left(r_{\max }\right)\right)^{1 / 2}$ in front of second term comes from periodicity matching of AdS black hole and thermally charged AdS geometries. Using this expression, we calculated grand potential, which is given as

$$
\begin{aligned}
\Delta \Omega=V_{d} \frac{1}{\kappa^{2}}\{( & \left.\left(r_{\mathrm{IR}}\right)^{d(\alpha+1)+1}-\left(r_{H}\right)^{d(\alpha+1)+1}\right) \\
& +\mu^{2}\{d(1+\alpha)-1\} e^{\lambda \phi_{0}} \\
& \left.\times\left(\frac{1}{4} r_{\mathrm{IR}}^{d(\alpha+1)-1}-r_{H}^{d(\alpha+1)-1}\right)\right\} .
\end{aligned}
$$

If the value of $\Delta \Omega$ is less than zero, the dominant geometry is AdS black hole and vice versa. The sign of $\Delta \Omega$ governs the nature of the stability of phase and confinement/deconfinement transition. The value of $r_{\mathrm{IR}}$ is set to be $323 \mathrm{MeV}$, which is calculated from the mass of lightest mesons [14]. We have plotted grand potential difference $(\Delta \Omega)$ versus temperature $(T)$ for various values of warp factor $\alpha$ which are given in Figure 1.

The relation describing the five-dimensional gravitational constant and that of the five-dimensional gauge coupling constant are evaluated by the application of AdS/CFT to QCD. These constants relates the colour gauge group $\left(N_{c}\right)$ and number of flavours $\left(N_{f}\right)$ as

$$
\frac{1}{2 \kappa^{2}}=\frac{N_{c}^{2}}{8 \pi^{2}}, \quad \frac{1}{2 g^{2}}=\frac{N_{c} N_{f}}{8 \pi^{2}} .
$$

In our study, we have used $N_{f}=2$ and $N_{c}=3$. 


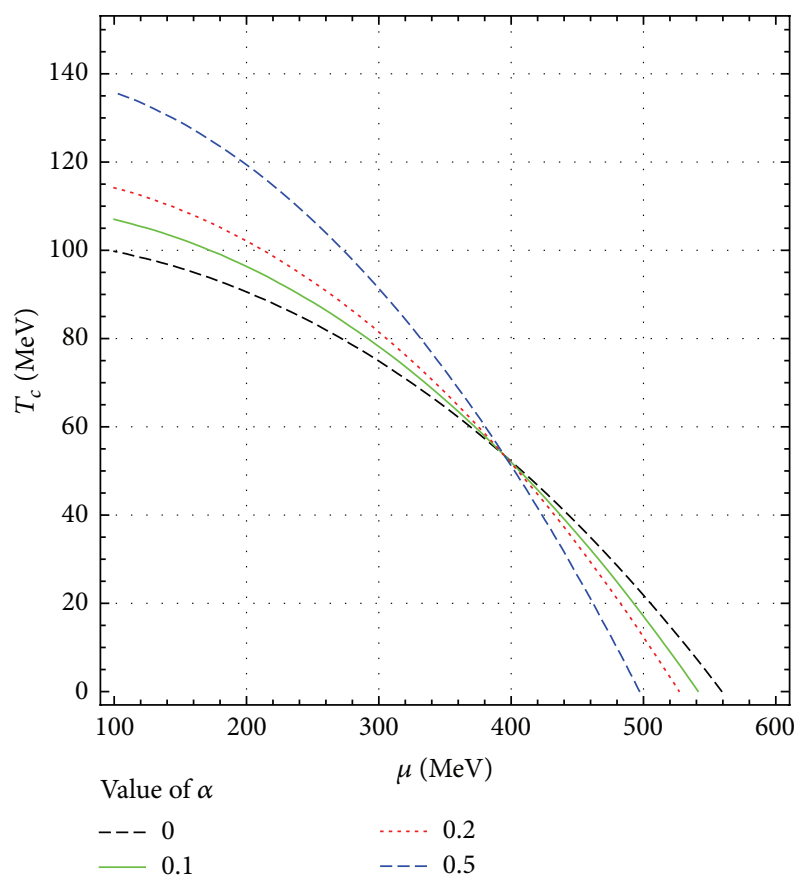

FIGURE 2: $T$ versus $\mu$ for various values of $\alpha$.

The value of $\alpha$ is considered to be equal to $-\theta / d$, which is commonly used in literature mentioned in [32]. We have taken dimension for our estimation to be five; that is, $d=3$ and $\phi_{0}=0$. To get plot $T$ versus $\mu$, we equate $\Delta \Omega=0$ and these plots for various values of $\alpha$ are given in Figure 2 .

\section{Conclusion}

In this paper, we studied the thermodynamic behavior of AdS/QCD from holographic approach with generalized warp factor. The plots of grand potential per unit volume are shown in Figure 1. Figure 1(a) shows grand potential per unit volume versus temperature for $\alpha=0.1$ and Figure 1(b) for $\alpha=$ 0.3 . These plots show that the increasing value of chemical potential $\mu$, for constant $\alpha$, and transition temperature got lowered but the maximum value of grand potential increases, which indicates the stability of thermally charged AdS at lower temperatures. The entropy difference $\Delta S$ (entropy is defined as $\left.S=-(\partial \Omega / \partial T)_{V, \mu}\right)$ is nonzero, which shows that the transition is of first order (using Ehrenfest scheme for classification of phase transition).

Figure 2 shows the plot between chemical potential and transition temperature for various values of $\alpha$. The results obtained here show similar qualitative behavior with various results obtained without warping dependence on dimension except the fact that, for different values of warping, all plots of Figure 2 meet at a point. It means that the transition is independent of warping on this point. We believe that it is the onset of second order transition. This is also expected from recent lattice data [33]. It would be interesting to study mass spectra of mesons in this scenario, transport properties, and corrections arising due to Gauss-Bonnet gravity.

\section{Conflict of Interests}

The author declares that there is no conflict of interests regarding the publication of this paper.

\section{Acknowledgments}

S. Sachan is supported by CSIR-Senior Research Fellowship, Grant no. (09/013(0239)/2009-EMR-I). The author would also like to thank Dr. Sanjay Siwach for discussing the problem at various stages of this work.

\section{References}

[1] J. B. Kogut and M. A. Stephanov, The Phases of Quantum Chromody-namics: From Confinement to Extreme Environments, Cambridge University Press, 2004.

[2] J. Maldacena, "The large $N$ limit of superconformal field theories and supergravity," Advances in Theoretical and Mathematical Physics, vol. 2, no. 2, pp. 231-252, 1998.

[3] E. Witten, "Anti-de Sitter space and holography," Advances in Theoretical and Mathematical Physics, vol. 2, no. 2, pp. 253-291, 1998.

[4] S. S. Gubser, I. R. Klebanov, and A. M. Polyakov, "Gauge theory correlators from non-critical string theory," Physics Letters B, vol. 428, no. 1-2, pp. 105-114, 1998.

[5] E. Witten, "Anti-de Sitter space, thermal phase transition, and confinement in gauge theories," Advances in Theoretical and Mathematical Physics, vol. 2, no. 3, pp. 505-532, 1998.

[6] A. Brandhuber, N. Itzhaki, J. Sonnenschein, and S. Yankielowicz, "Wilson loops, confinement, and phase transitions in large $N$ gauge theories from supergravity," The Journal of High Energy Physics, vol. 1998, no. 6, article 001, 1998.

[7] J. Erlich, E. Katz, D. T. Son, and M. A. Stephanov, "QCD and a holographic model of hadrons," Physical Review Letters, vol. 95, no. 26, Article ID 261602, 2005.

[8] A. Karch, E. Katz, D. T. Son, and M. A. Stephanov, "Linear confinement and AdS/QCD," Physical Review D, vol. 74, no. 1, Article ID 015005, 2006.

[9] C. A. Ballon Bayona, H. Boschi-Filho, N. R. F. Braga, and L. A. Pando Zayas, "On a holographic model for connement/deconfinement," Physical Review D, vol. 77, Article ID 046002, 2008.

[10] E. Megías, H. J. Pirner, and K. Veschgini, "QCD thermodynamics using five-dimensional gravity," Physical Review D, vol. 83, no. 5, Article ID 056003, 2011.

[11] K. Veschgini, E. Megías, and H. J. Pirner, "Trouble finding the optimal AdS/QCD," Physics Letters B, vol. 696, no. 5, pp. 495498, 2011.

[12] L. Da Rold and A. Pomarol, "Chiral symmetry breaking from five-dimensional spaces," Nuclear Physics B, vol. 721, no. 1-3, pp. 79-97, 2005.

[13] A. Parnachev and D. A. Sahakyan, "Chiral phase transition from string theory," Physical Review Letters, vol. 97, no. 11, Article ID 111601, 4 pages, 2006.

[14] C. P. Herzog, "A holographic prediction of the deconfinement temperature," Physical Review Letters, vol. 98, Article ID 091601, 2007.

[15] T. Gherghetta, J. I. Kapusta, and T. M. Kelley, "Chiral symmetry breaking in the soft-wall AdS/QCD model," Physical Review D, vol. 79, Article ID 076003, 2009. 
[16] J. Erdmenger, N. Evans, I. Kirsch, and E. J. Threlfall, "Mesons in gauge/gravity duals," European Physical Journal A, vol. 35, no. 1, pp. 81-133, 2008.

[17] R. Cai and J. P. Shock, "Holographic confinement/deconfinement phase transitions of AdS/QCD in curved spaces," Journal of High Energy Physics, vol. 2007, no. 8, article 095, 2007.

[18] O. Andreev, "Cold quark matter, quadratic corrections, and gauge/string duality," Physical Review D, vol. 81, no. 8, Article ID 087901, 2010.

[19] C. Park, D.-Y. Gwak, B.-H. Lee, Y. Ko, and S. Shin, "Soft wall model in the hadronic medium," Physical Review D, vol. 84, Article ID 046007, 2011.

[20] S. Sachan and S. Siwach, "Thermodynamics of soft wall AdS/QCD at finite chemical potential," Modern Physics Letters A, vol. 27, no. 28, Article ID 1250163, 2012.

[21] B.-H. Lee, C. Park, and S.-J. Sin, "A dual geometry of the hadron in dense matter," Journal of High Energy Physics, vol. 7, article 087, 2009.

[22] P. Zhang, "Linear confinement for mesons and nucleons in AdS/QCD," Journal of High Energy Physics, vol. 2010, no. 5, article 039, 2010.

[23] D. K. Hong, T. Inami, and H. Yee, "Baryons in AdS/QCD," Physics Letters B: Nuclear, Elementary Particle and High-Energy Physics, vol. 646, no. 4, pp. 165-171, 2007.

[24] T. Sakai and S. Sugimoto, "Low energy hadron physics in holographic QCD," Progress of Theoretical Physics, vol. 113, pp. 843-882, 2005.

[25] T. Sakai and S. Sugimoto, "More on a holographic dual of QCD," Progress of Theoretical Physics, vol. 114, no. 5, pp. 1083-1118, 2005.

[26] S. W. Hawking and D. N. Page, "Thermodynamics of black holes in anti-de Sitter space," Communications in Mathematical Physics, vol. 87, no. 4, pp. 577-588, 1982/83.

[27] Y. Kim, B.-H. Lee, S. Nam, C. Park, and S.-J. Sin, "Deconfinement phase transition in holographic QCD with matter," Physical Review D, vol. 76, Article ID 086003, 2007.

[28] M. Cvetič, S. Nojiri, and S. D. Odintsov, "Black hole thermodynamics and negative entropy in de Sitter and anti-de Sitter Einstein-Gauss-Bonnet gravity," Nuclear Physics B, vol. 628, no. 1-2, pp. 295-330, 2002.

[29] B. S. Kim, "Hyperscaling violation: a unified frame for effective holographic theories," Journal of High Energy Physics, vol. 2012, no. 11, article 061, 2012.

[30] X. Dong, S. Harrison, S. Kachru, G. Torroba, and H. Wang, "Aspects of holography for theories with hyperscaling violation," Journal of High Energy Physics, vol. 2012, article 41, 2012.

[31] J. Gath, J. Hartong, R. Monteiro, and N. A. Obers, "Holographic models for theories with hyperscaling violation," Journal of High Energy Physics, vol. 2013, no. 4, article 159, 2013.

[32] M. Alishahiha, E. O'Colgain, and H. Yavartanoo, "Charged Black Branes with Hyperscaling Violating Factor," Journal of High Energy Physics, vol. 2012, article 137, 2012.

[33] M. Fromm, J. Langelage, S. Lottini, and O. Philipsen, "The QCD deconfinement transition for heavy quarks and all baryon chemical potentials," Journal of High Energy Physics, vol. 2012, no. 1, article 042, 2012. 

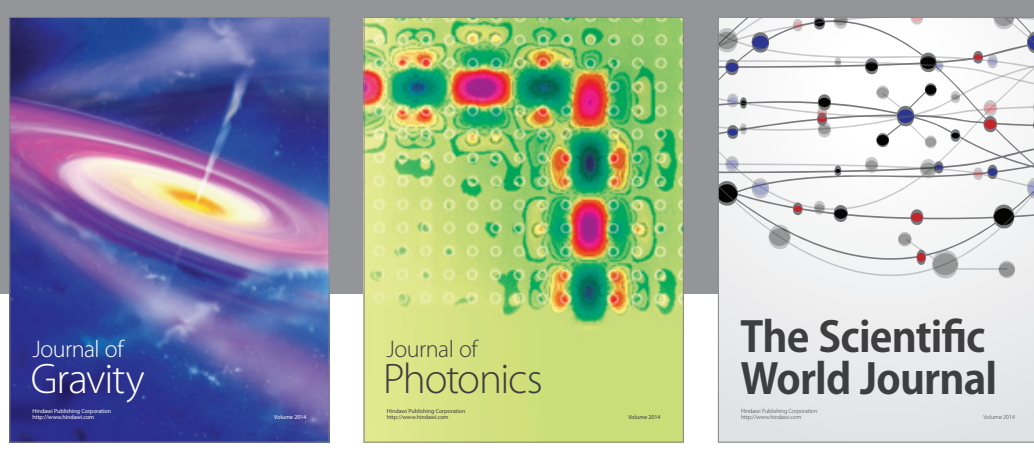

The Scientific World Journal
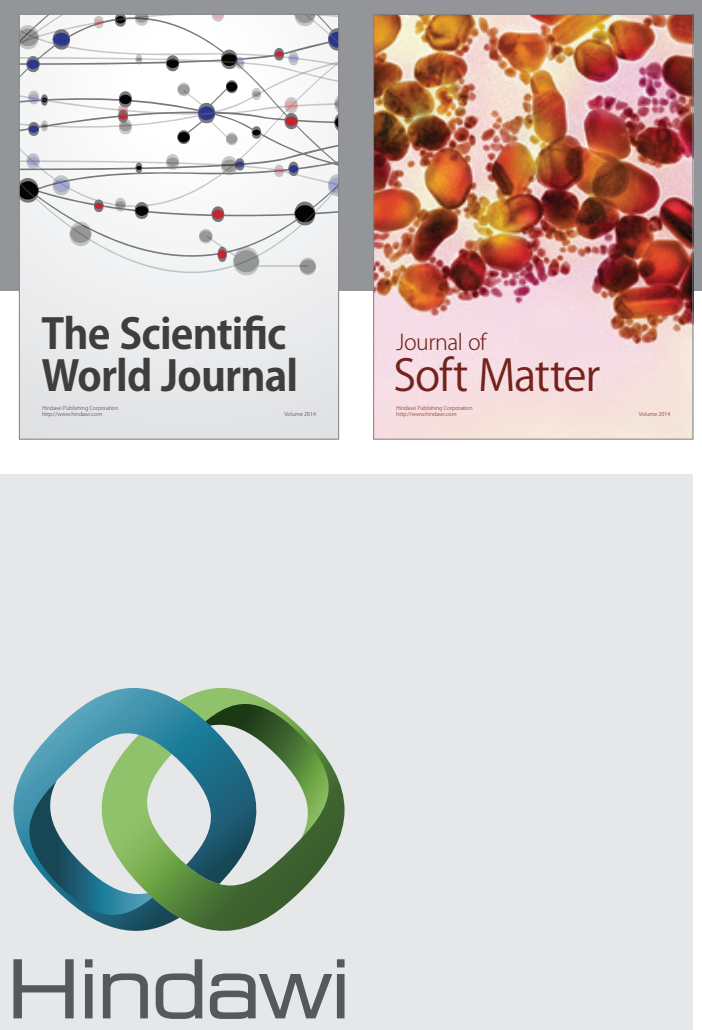

Submit your manuscripts at

http://www.hindawi.com

nternational Journal of

Statistical Mechanics
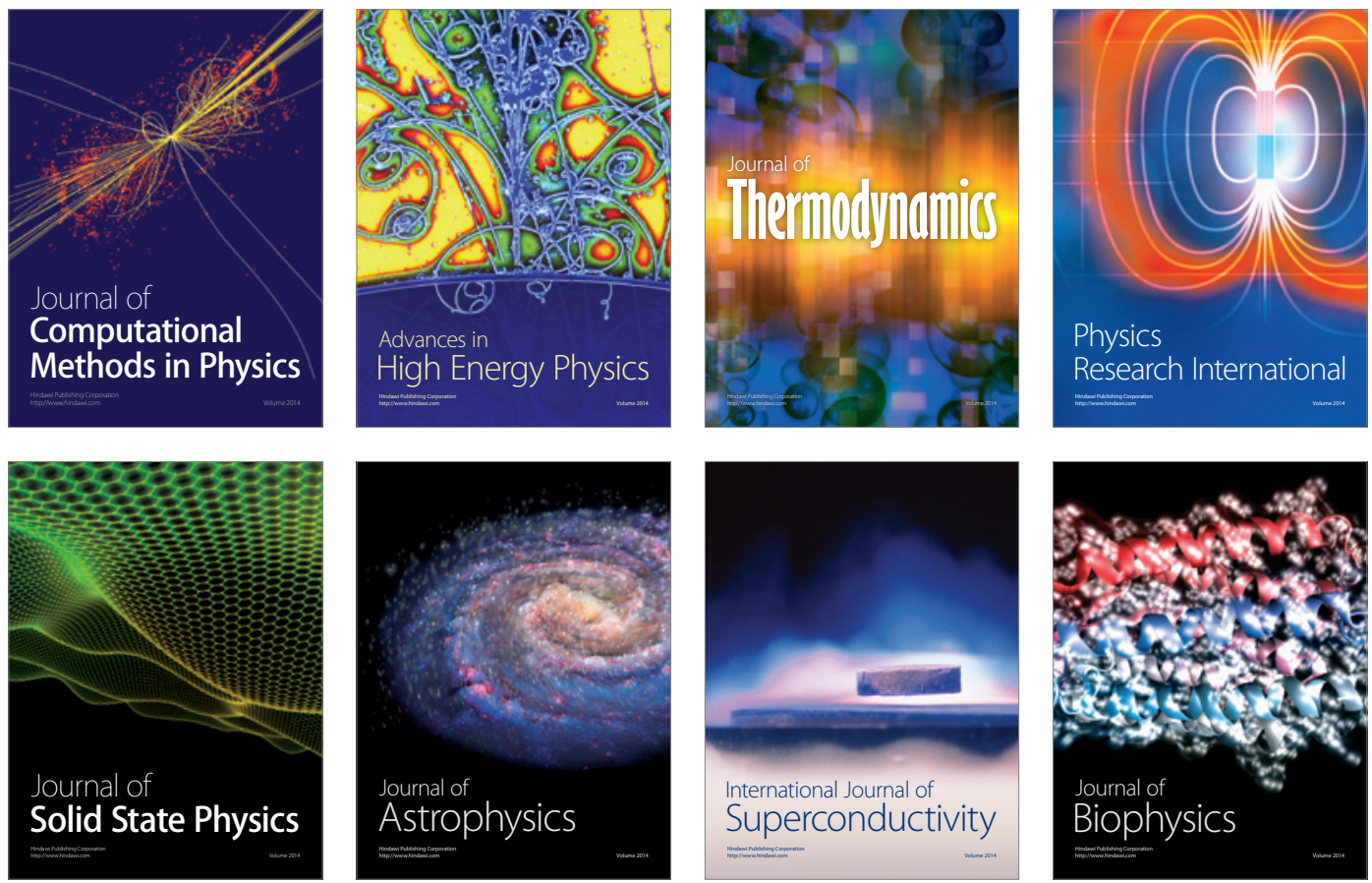
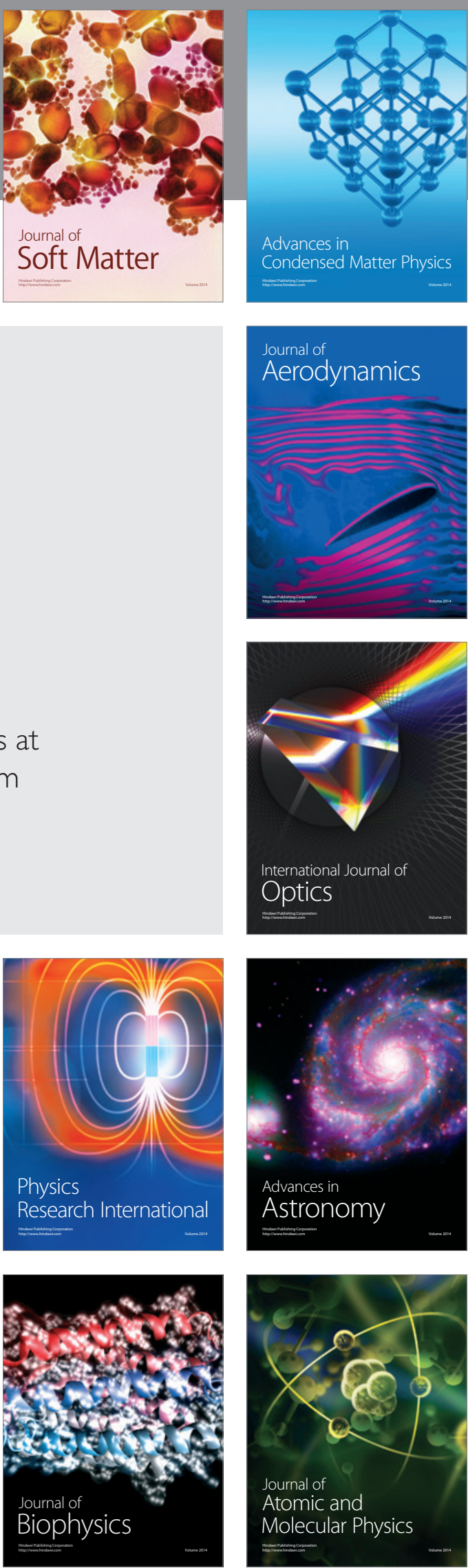